\title{
Reference Values for Shear Wave Elastography of Neck and Shoulder Muscles in Healthy Individuals
}

\section{(ㄷ) (1) (요 $\Theta$}

\author{
Authors \\ Caroline Ewertsen ${ }^{1}$, Jonathan Carlsen ${ }^{2}$, Mohammed Aftab Perveez ${ }^{3}$, Henrik Schytz ${ }^{3}$
}

Affiliations

1 Copenhagen University Hospital, Rigshospitalet, Department of Radiology, Copenhagen OE, Denmark

2 Rigshospitalet, Radiologisk klinik, Kopenhagen, Denmark

3 Rigshospitalet, Glostrup, Headache Diagnostic Laboratory, Danish Headache Center and Department of Neurology, Faculty of Health and Medical Sciences, University of Copenhagen, Glostrup, Denmark

Key words

muscular, ultrasound, elastography

$\begin{array}{lr}\text { received } & 14.04 .2017 \\ \text { revised } & 25.10 .2017 \\ \text { accepted } & 09.01 .2018\end{array}$

Bibliography

DOI https://doi.org/10.1055/s-0044-102013

Ultrasound Int Open 2018; 4: E23-E29

(c) Georg Thieme Verlag KG Stuttgart · New York

ISSN 2199-7152

Correspondence

Dr. Caroline Ewertsen, MD, Phd

Copenhagen University Hospital, Rigshospitalet

Department of Radiology

Blegdamsvej 9

Copenhagen OE, DK-2100

Denmark

Tel.: + 45/354/53 419, Fax: + 45/354/52 058

caroline.ewertsen@dadlnet.dk

\begin{abstract}
Purpose to establish reference values for ultrasound shearwave elastography for pericranial muscles in healthy individuals (m. trapezius, m. splenius capitis, m. semispinalis capitis, m. sternocleidomastoideus and $\mathrm{m}$. masseter). Also to evaluate day-to-day variations in the shear-wave speeds and evaluate the effect of the pennation of the muscle fibers, ie scanning parallel or perpendicularly to the fibers.
\end{abstract}

Materials and Methods 10 healthy individuals ( 5 males and 5 females) had their pericranial muscles examined with shearwave elastography in two orthogonal planes on two different days for their dominant and non-dominant side. Mean shear wave speeds from 5 ROl's in each muscle, for each scan plane for the dominant and non-dominant side for the two days were calculated. The effect of the different parameters - muscle pennation, gender, dominant vs non-dominant side and day was evaluated.

Results The effect of scan plane in relation to muscle pennation was statistically significant $(p<0.0001)$. The mean shearwave speed when scanning parallel to the muscle fibers was significantly higher than the mean shear-wave speed when scanning perpendicularly to the fibers. The day-to-day variation was statistically significant $(p=0.0258)$, but not clinically relevant. Shear-wave speeds differed significantly between muscles. Mean shear wave speeds $(\mathrm{m} / \mathrm{s})$ for the muscles in the parallel plane were: for masseter 2.45 (SD: $+\mid-0.25)$, semispinal 3.36 (SD: + / - 0.75), splenius 3.04 (SD: + | - 0.65), sternocleidomastoid 2.75 (SD: + / - 0.23), trapezius 3.20 (SD: + / - 0.27) and trapezius lateral 3.87 (SD: + / -3.87).

Conclusion The shear wave speed variation depended on the direction of scanning. Shear wave elastography may be a method to evaluate muscle stiffness in patients suffering from chronic neck pain.

\section{Introduction}

Ultrasound (US) elastography is a method for evaluating tissue stiffness. Mainly two different methods are commercially available strain and shear wave elastography. Shear wave elastography can be subdivided into shear wave speed measurements and shear wave speed imaging according to the EFSUMB guidelines [1].
Strain elastography has mainly been evaluated for focal lesions in the breast and in the thyroid [2,3] and is based on mechanical stress applied by the physician performing the examination. The elasticity is shown visually on a color scale from blue to red and semiquantitative values may be calculated. Shear wave elastography evaluates tissue stiffness by applying an acoustic push pulse, 
which deforms the tissue. This deformation causes shear waves, which occur perpendicularly to the US push pulse. The speed of these shear waves is proportional to tissue stiffness and a quantitative value of stiffness is shown. The method has mainly been evaluated for assessment of liver stiffness but also recently in skeletal muscle $[4,5]$.

Tension-type headache (TTH) is subdivided into tension-type headache associated with or without pericranial muscle tenderness [6] and the one-year prevalence of neck pain is $88 \%$ in TTH [7]. Experimentally, pericranial muscles are significantly more tender in patients with chronic tension-type headache than in healthy controls [8] and the degree of tenderness is strongly correlated to the frequency and intensity of tension-type headaches [9]. In addition, pericranial muscles are significantly harder in patients with chronic tension-type headache than in healthy controls [10], which correlate with muscle tenderness of the trapezius muscle and relate to the headache state [11]. To date, there are no studies using elastography in primary headaches, but US elastography may be an ideal noninvasive quantitative tool to uncover muscle tension in these patients.

The aim of the study was to establish reference values for shear wave elastography for the pericranial muscles in healthy individuals, more specifically the m. trapezius, $m$. splenius capitis, m. semispinalis capitis, $m$. sternocleidomastoideus and m. masseter, and to evaluate day-to-day variations in shear wave speeds and evaluate the effect of the pennation of the muscle fibers, i.e., scanning parallel or perpendicular to the fibers.

\section{Materials and Methods}

\section{Subjects}

All subjects gave informed oral consent to participate in the study. The study was approved by the Regional Committee on Health Research Ethics (ID: H-15014237). 10 healthy volunteers were included in the study - 5 males and 5 females, median age: 31 years (range: 22-46 years). All subjects were physically active, but were asked not to do any sports within $48 \mathrm{~h}$ before the study. The median weight, height and BMI were $70.5 \mathrm{~kg}$ (range: $48-105 \mathrm{~kg}$ ), $177 \mathrm{~cm}$ (range: 157-195 cm) and 22.4 (range: 19.5-27.6), respectively. All volunteers were scanned twice with one week separating the two sessions in order to determine possible day-to-day variation.

The volunteers had the trapezius muscle, the semispinalis capitis muscle, the splenius muscle, the sternocleidoid muscle and the

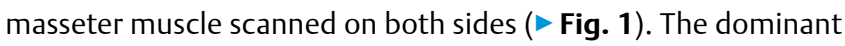
hand and possible sport activity were registered.

\section{Ultrasound}

A GE Logic E9 system with a $9 \mathrm{MHz}$ linear array transducer and MSK preset (GE, Chalfont St. Giles) was used for all measurements. All muscles were scanned parallel and perpendicular to the direction of the muscle fibers. For the trapezius, two measurements, one medial measurement and one more lateral measurement, were performed. In the masseter muscle two scan planes perpendicular to each other were obtained, but due to the pennation of the muscle it was impossible to obtain images with only one direction of the pennation.

\section{Elastography}

The system shows real-time color coded shear-wave elastography images in a pre-defined $\mathrm{ROI}$ of the $\mathrm{B}$-mode image with a frame rate of approximately $1 \mathrm{~Hz}$. Cine loops of ten frames were stored in the system for later post-processing. All recordings were performed by the same experienced physician with light pre-compression on the skin. The subjects were examined sitting on a chair with their arms resting in their lap. All recordings were done from the back of the patient. An example of an elastogram is shown in - Fig. 2-4.

After all recordings were performed, the shear wave speeds of the different muscles in $\mathrm{m} / \mathrm{s}$ were registered. ROIs were placed in each muscle in agreement by two observers. Circular ROIs were placed to cover as much muscle as possible. Five measurements were performed in each cine loop from each measuring point in

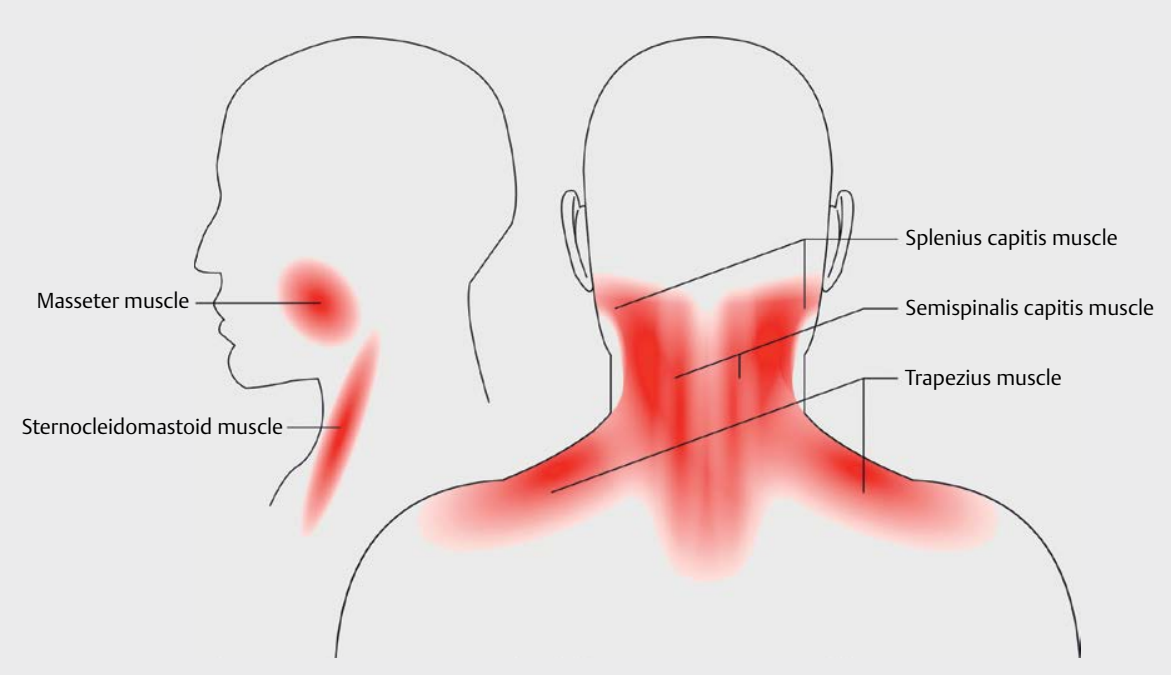

- Fig. 1 Drawing of the muscles examined in two planes (Thien Phu Do is acknowledged for drawing the figure) 


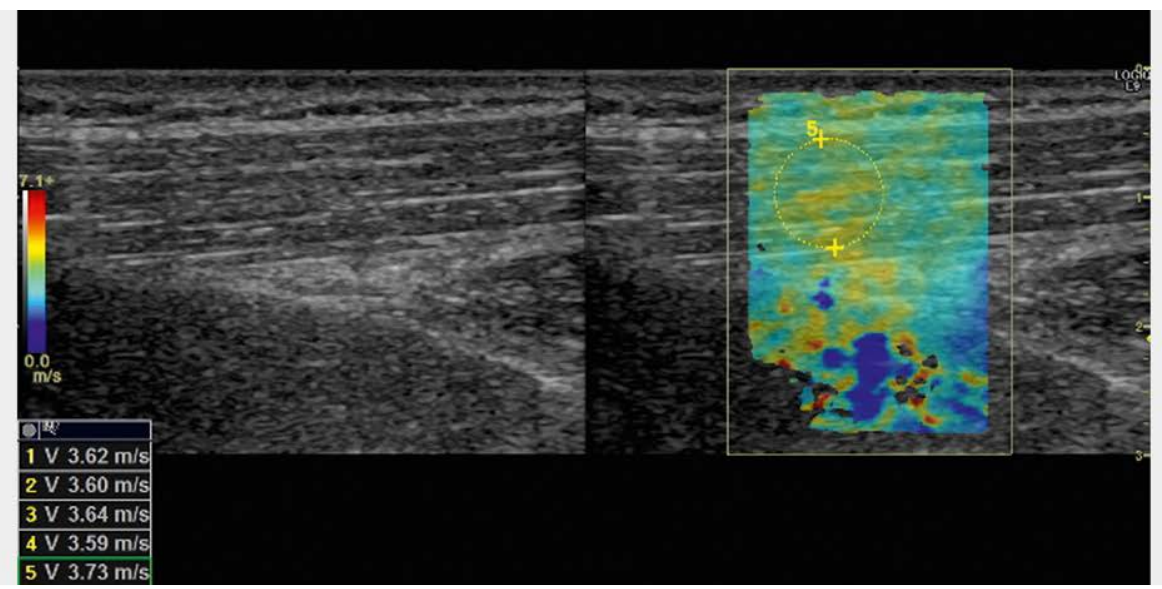

- Fig. 2 B-mode image of the trapezius muscle (left) and corresponding image with elastogram (right). Shear wave speed is shown in the lower left corner for 5 different ROls (only number 5 shown).

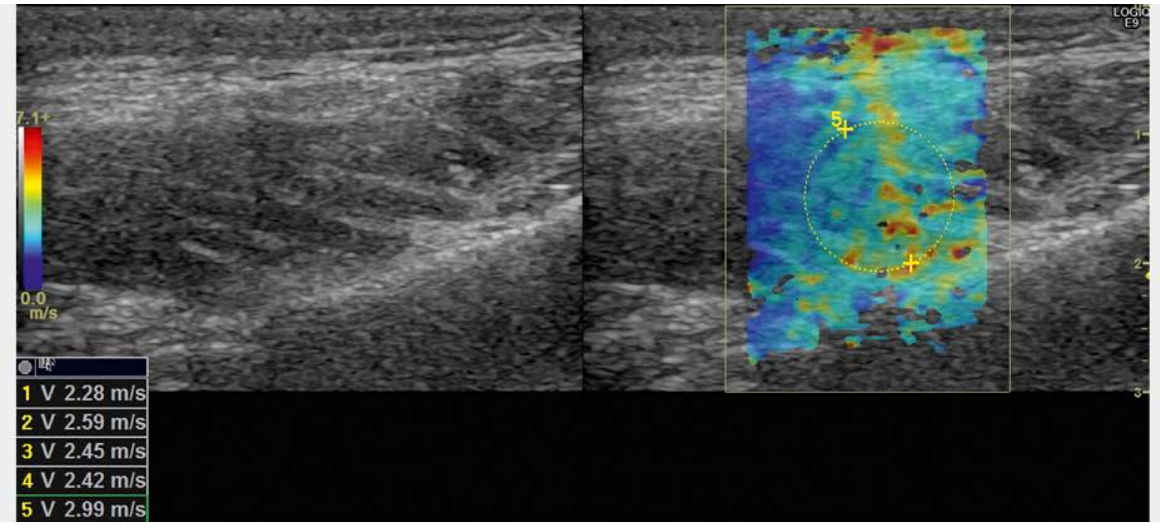

- Fig. 3 B-mode image of the masseter muscle (left) and corresponding image with elastogram (right)

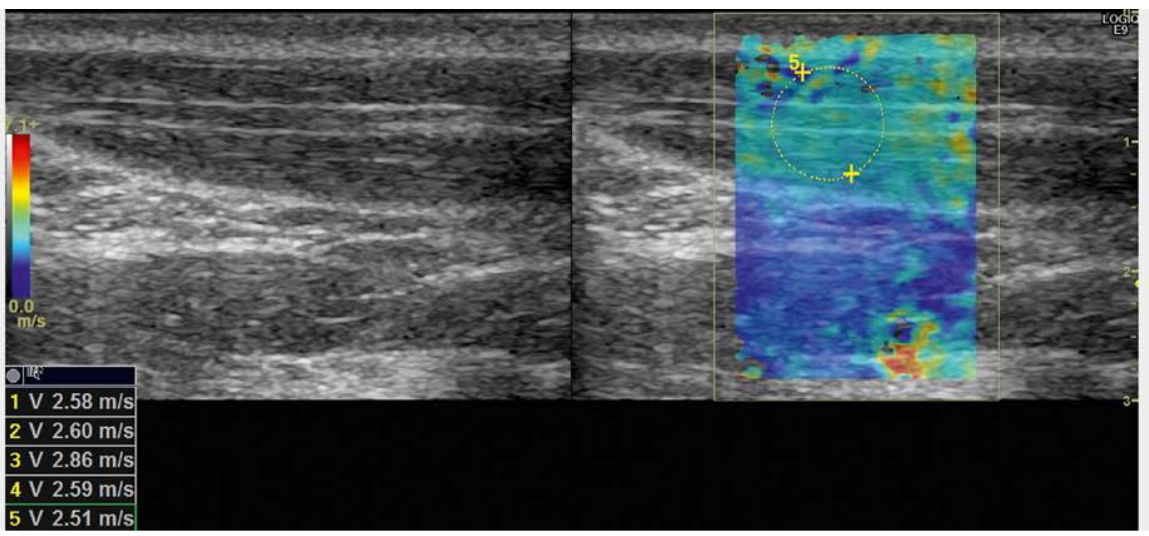

- Fig. 4 B-mode image of the sternocleidomastoid muscle (left) and corresponding image with elastogram (right)

each muscle - one for each recorded frame. The size and depth of the ROI were registered for all measurements.

\section{Statistics}

The mean shear wave speeds for all muscle groups in the two orthogonal planes were calculated.

The repeated measurements were analyzed in a mixed effects linear statistical model including sequence, side, dominant side, muscle group, gender, muscle group-scanning direction interaction, dominant side-scanning direction interaction, sequence-scanning direction, and sequence-muscle group interaction as fixed effects and subject-dominant side-muscle group-scanning direction as random effect.

The effects of sequence, dominant side and scanning direction were tested in the statistical model using SAS 9.4 (SAS Institute). The fixed effects were tested in the full model using two-sided type 


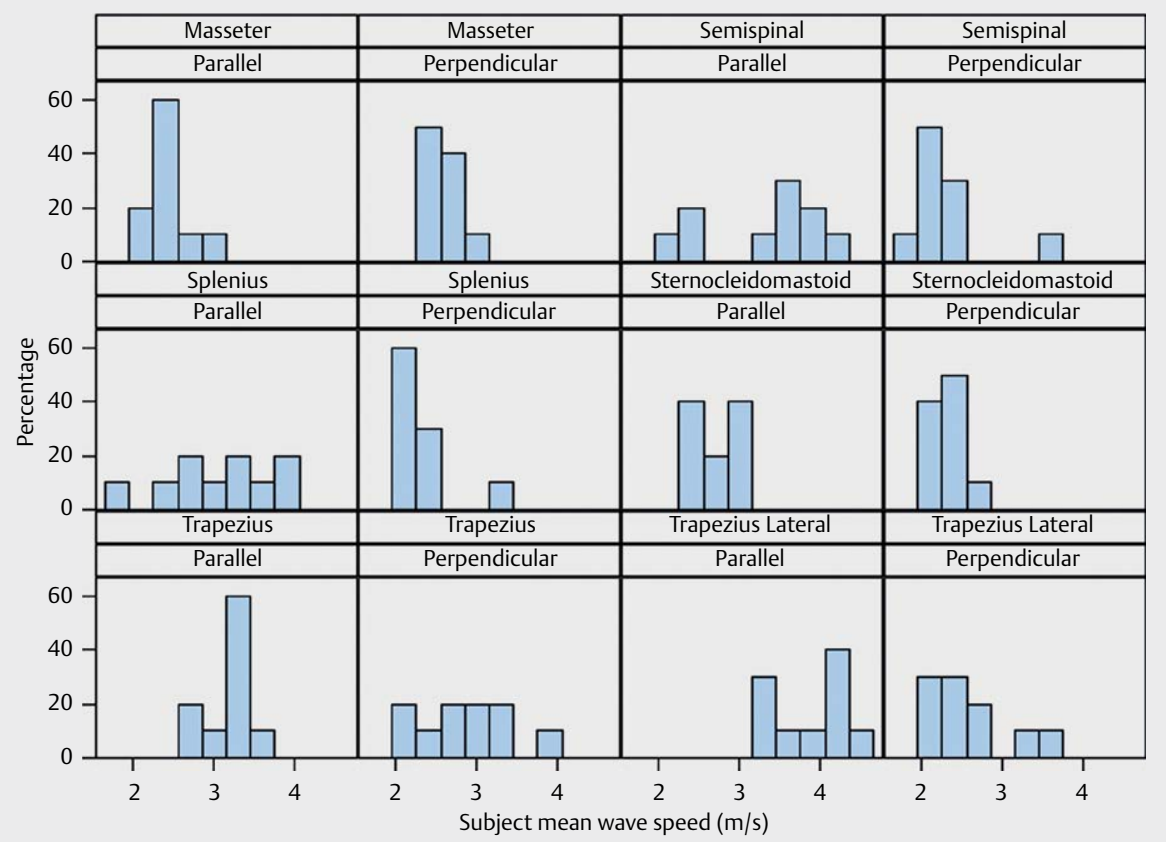

Fig. 5 Probability histogram panel plot. X-axis shows mean shear wave speed and y-axis shows proportion of subjects for all examined muscles in two planes.

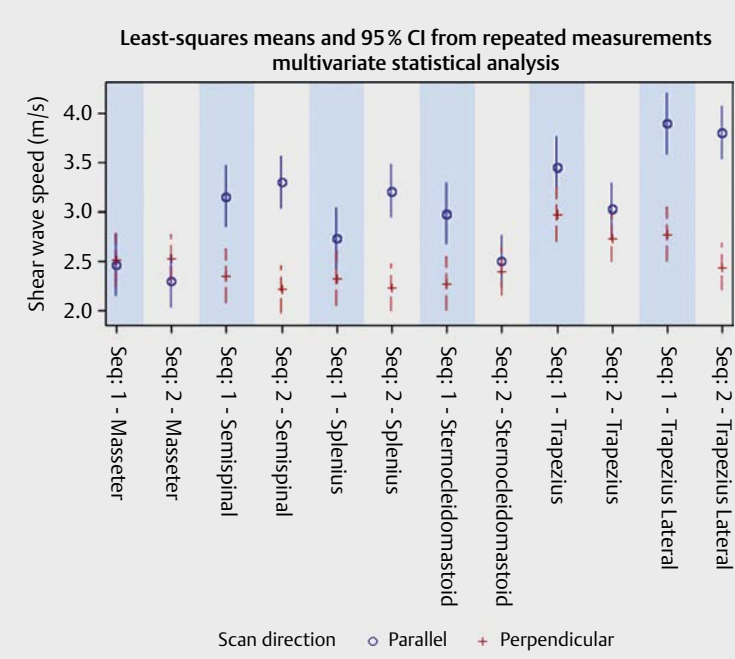

The mean wave speed is analysed, for each scan direction, in a repeated multivariate linear statistical model including sequence-muscle interaction as fixed effects and 5 repetitions on two different days as repeated measurments.

- Fig. 6 Mean shear wave speed for all examined muscles for each scan plane

3 tests of effects. A paired t-test was performed to analyze the variation between days.

\section{Results}

A total of 20 repeated samples were measured for each of the 6 groups of muscles for each subject for right and left sides, adding up to 240 measurements per subject. The mean shear wave speeds $(\mathrm{m} / \mathrm{s})$ in the parallel plane were: $2.45(\mathrm{SD}:+/-0.25)$ for masseter, 3.36 (SD: + / - 0.75) for semispinalis, 3.04 (SD: + / - 0.65) for splenius, 2.75 (SD: + / - 0.23) for sternocleidomastoid, 3.20 (SD: + / - 0.27) for trapezius and 3.87 (SD: + / - 3.87) for trapezius lateral. $>$ Fig. 5 presents histograms for the combination of muscle groups and scanning direction using subject mean values within each category.

The effect of sequence (day-to-day variation) was statistically significant ( $p=0.0258$, test for sequence-muscle group interaction). $>$ Fig. 6 shows that even though the effect is statistically significant, the effect is not in the same direction for all muscle groups. Further analysis showed no statistically significant difference between the two days. The mean shear wave speed for each muscle, each fiber direction, dominant and non-dominant hand for the two different days is shown in $>$ Table 1.

The effect of dominant side was not statistically significant ( $p=0.69$, test for dominant side-scanning direction interaction and $p=0.60$ main effect of dominant side).

The effect of scan plane in relation to muscle pennation was statistically significant $(p<0.0001$, test for muscle-scanning direction interaction). The mean shear-wave speed when scanning parallel to the muscle fibers was significantly higher than the mean shearwave speed when scanning perpendicular to the fibers. The results are shown as model estimate LSMeans in > Fig. 7. Values differed significantly between muscles.

\section{Discussion}

We have examined factors influencing the elasticity of head and neck muscles in healthy individuals and found that the pennation 
- Table 1 Mean shear wave speed for each muscle, each fiber direction, dominant and non-dominant hand for each of the two days including SD.

\begin{tabular}{|c|c|c|c|c|c|}
\hline Dominant side & Muscle & Fiber direction & Day & Mean $(\mathrm{m} / \mathrm{s})$ & SD \\
\hline Yes & Masseter & Parallel & 1 & 2.38 & 0.31 \\
\hline Yes & & & 2 & 2.38 & 0.26 \\
\hline Yes & & Perpendicular & 1 & 2.49 & 0.35 \\
\hline Yes & & & 2 & 2.48 & 0.41 \\
\hline Yes & Semispinalis & Parallel & 1 & 3.50 & 1.15 \\
\hline Yes & & & 2 & 3.67 & 1.15 \\
\hline Yes & & Perpendicular & 1 & 2.36 & 0.47 \\
\hline Yes & & & 2 & 2.09 & 0.53 \\
\hline Yes & Splenius & Parallel & 1 & 2.78 & 0.57 \\
\hline Yes & & & 2 & 3.30 & 1.08 \\
\hline Yes & & Perpendicular & 1 & 2.44 & 0.51 \\
\hline Yes & & & 2 & 2.18 & 0.53 \\
\hline Yes & Sternocleidomastoid & Parallel & 1 & 3.14 & 0.39 \\
\hline Yes & & & 2 & 2.53 & 0.30 \\
\hline Yes & & Perpendicular & 1 & 2.29 & 0.54 \\
\hline Yes & & & 2 & 2.37 & 0.30 \\
\hline Yes & Trapezius & Parallel & 1 & 3.32 & 0.60 \\
\hline Yes & & & 2 & 2.80 & 0.37 \\
\hline Yes & & Perpendicular & 1 & 3.04 & 0.73 \\
\hline Yes & & & 2 & 3.03 & 0.72 \\
\hline Yes & Trapezius lateral & Parallel & 1 & 3.93 & 0.65 \\
\hline Yes & & & 2 & 3.71 & 0.41 \\
\hline Yes & & Perpendicular & 1 & 2.82 & 1.03 \\
\hline Yes & & & 2 & 2.54 & 0.29 \\
\hline No & Masseter & Parallel & 1 & 2.62 & 0.55 \\
\hline No & & & 2 & 2.43 & 0.37 \\
\hline No & & Perpendicular & 1 & 2.70 & 0.53 \\
\hline No & & & 2 & 2.51 & 0.31 \\
\hline No & Semispinalis & Parallel & 1 & 3.07 & 0.84 \\
\hline No & & & 2 & 3.22 & 0.58 \\
\hline No & & Perpendicular & 1 & 2.24 & 0.58 \\
\hline No & & & 2 & 2.33 & 0.63 \\
\hline No & Splenius & Parallel & 1 & 2.93 & 1.02 \\
\hline No & & & 2 & 3.15 & 0.48 \\
\hline No & & Perpendicular & 1 & 2.13 & 0.43 \\
\hline No & & & 2 & 2.40 & 0.69 \\
\hline No & Sternocleidomastoid & Parallel & 1 & 2.82 & 0.47 \\
\hline No & & & 2 & 2.50 & 0.30 \\
\hline No & & Perpendicular & 1 & 2.24 & 0.38 \\
\hline No & & & 2 & 2.39 & 0.63 \\
\hline No & Trapezius & Parallel & 1 & 3.42 & 0.78 \\
\hline No & & & 2 & 3.25 & 0.49 \\
\hline No & & Perpendicular & 1 & 2.88 & 0.80 \\
\hline No & & & 2 & 2.43 & 0.31 \\
\hline No & Trapezius lateral & Parallel & 1 & 3.91 & 0.65 \\
\hline No & & & 2 & 3.93 & 0.62 \\
\hline No & & Perpendicular & 1 & 2.75 & 0.68 \\
\hline No & & & 2 & 2.33 & 0.60 \\
\hline
\end{tabular}




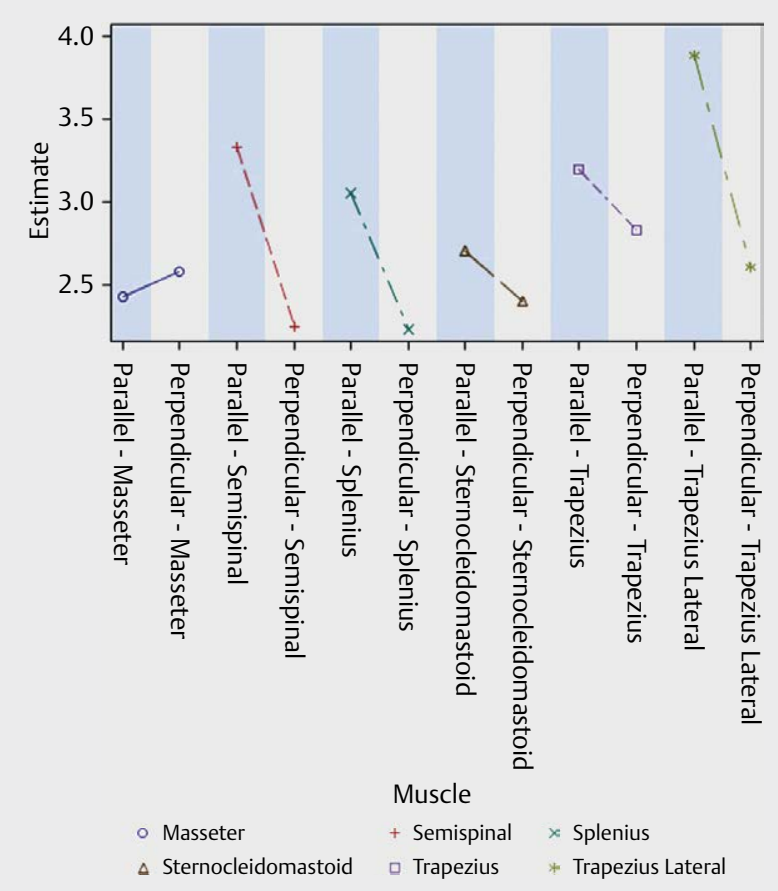

- Fig. 7 Mean shear wave speed estimates when scanning parallel and perpendicular to the muscle fibers

of the muscle fibers had an influence on the shear wave speeds measured.

This has been confirmed in other studies. Gennisson et al. examined beef muscle fibers and found that the shear waves propagated more easily parallel to the muscle fibers that perpendicular to them and Eby et al. examined shear moduli with increasing strain with different transducer positions - parallel, perpendicular and 45 degrees - and shear waves propagated better in the parallel position $[12,13]$.

The shear modulus of tissue may be calculated from the shearwave speed using the equation: $\mu=c^{2} \rho$, where $\mu$ is the shear modulus, $c$ is the shear wave speed and $\rho$ is the density which may be assumed to be $1000 \mathrm{~kg} / \mathrm{m}^{3}$. This formula assumes isotropy, and homogeneous materials, and it may only be accurate to report shear modulus when the waves are propagating parallel to the underlying muscle fibers. In other transducer orientations it may be more correct only to report shear wave speeds $[1,13]$. We only reported the shear wave speeds in order to be able to compare the values from scanning parallel and perpendicular to the muscle fibers. Also in the masseter muscle fibers were interleaved in different directions. In musculoskeletal US, anisotropy is a well-known artifact especially when scanning tendons. Skeletal muscle may be considered transversely isotropic but in this transducer orientation anisotropy from the examined tissue may be a challenge [14].

There was a statistically significant effect from the day the volunteers were scanned, but we do not find it clinically relevant and it may be due to coincidence in this relatively small population, also because the effect was not in the same direction for all muscles. Niitsu et al. found a post-exercise hardness of the biceps muscle, which lasted for two to four days [15]. All volunteers were asked not to perform vigorous exercise two days before our measurements. This may also explain why we did not find a statistically significant effect of dominant vs. non-dominant hand. Furthermore, the muscles were examined when relaxed and not during exercise.

Another group has examined US elastography for evaluation of trigger points [16]. Although they only examined three persons with chronic neck pain and compared them with 17 healthy volunteers, they found significantly stiffer trapezius muscles in the persons suffering from chronic neck pain. They examined the muscle in the transverse plane and the median shear wave speed was $2.02+/-0.42 \mathrm{~m} / \mathrm{s}$ in healthy volunteers and $2.49+/-0.39$ in persons with chronic neck pain. Our mean values in the trapezius medial and lateral were: $2.84+/-0.55 \mathrm{~m} / \mathrm{s}$ and $2.61+/-0.49 \mathrm{~m} / \mathrm{s}$, respectively, where the medial position was comparable to the position in the paper. This difference may be due to the elastography method not being exactly the same. We measured shear wave speeds from an area sampled in real time and the authors of the other study used point shear wave elastography from another vendor.

We chose the ROI to cover as much as possible of the muscle thickness to obtain representative values. We noticed that the signal was dampened in persons with thick muscles and in the muscles that were lying deeper (semispinalis muscle compared to splenius muscle). Other studies have shown a dependency on depth in a phantom and in the liver for shear wave elastography [17] and also in muscles [18]. Kot et al. found an effect of ROI size on the maximum value of the shear wave speed, but not on the mean shear wave speed when scanning the thigh muscles [19].

\section{Limitations}

Our study had some limitations. Although the same radiologist examined the volunteers, it was impossible to ensure that the measurement was performed at exactly the same point in the two sessions. It was difficult to distinguish between the splenius and the semispinalis muscle, which were both rather thin muscles. Also the scan planes in the masseter were not only parallel or perpendicular to the fibers as the two heads of the masseter muscles are interleaved. All persons were examined in the sitting position resting, but even minor tension in the muscles may have influenced the measurements.

\section{Conclusion}

The shear wave speed variation depended on the direction of scanning. No difference was seen between measurements between dominant and non-dominant side. The day-to-day variation was statistically significant, but the differences were not clinically relevant.

Shear wave elastography may be a method to evaluate muscle stiffness in patients suffering from chronic neck pain.

\section{References}

[1] Bamber ], Cosgrove D, Dietrich CF, Fromageau J, Bojunga J, Calliada F et al. EFSUMB guidelines and recommendations on the clinical use of ultrasound elastography. Part 1: Basic principles and technology. Ultraschall in Med 2013; 34: 169-184 
[2] Cantisani V, D'Andrea V, Biancari F, Medvedyeva O, Di Segni M, Olive M et al. Prospective evaluation of multiparametric ultrasound and quantitative elastosonography in the differential diagnosis of benign and malignant thyroid nodules: Preliminary experience. Eur J Radiol 2012; 81: 2678-2683

[3] Itoh A, Ueno E, Tohno E, Kamma H, Takahashi H, Shiina T et al. Breast disease: Clinical application of US elastography for diagnosis. Radiology 2006; 239: 341-350

[4] Cassinotto C, Lapuyade B, Ait-Ali A, Vergniol J, Gaye D, Foucher J et al. Liver fibrosis: Noninvasive assessment with acoustic radiation force impulse elastography-comparison with FibroScan $\mathrm{M}$ and $\mathrm{XL}$ probes and FibroTest in patients with chronic liver disease. Radiology 2013; 269: 283-292

[5] Chino K, Akagi R, Dohi M, Fukashiro S, Takahashi H. Reliability and validity of quantifying absolute muscle hardness using ultrasound elastography. PloS One 2012; 7: e45764

[6] Headache Classification Committee of the International Headache $S$. The international classification of headache disorders, 3rd ed. (beta version). Cephalalgia 2013; 33: 629-808

[7] Ashina S, Bendtsen L, Lyngberg AC, Lipton RB, Hajiyeva N, Jensen R. Prevalence of neck pain in migraine and tension-type headache: A population study. Cephalalgia 2015; 35: 211-219

[8] Langemark M, Jensen K, Jensen TS, Olesen J. Pressure pain thresholds and thermal nociceptive thresholds in chronic tension-type headache. Pain 1989; 38: 203-210

[9] Jensen R, Rasmussen BK, Pedersen B, Olesen J. Muscle tenderness and pressure pain thresholds in headache. A population study. Pain 1993; 52: 193-199

[10] Sakai F, Ebihara S, Akiyama M, Horikawa M. Pericranial muscle hardness in tension-type headache. A non-invasive measurement method and its clinical application. Brain 1995; 118: 523-531
[11] Ashina M, Bendtsen L, Jensen R, Sakai F, Olesen J. Muscle hardness in patients with chronic tension-type headache: Relation to actual headache state. Pain 1999; 79: 201-205

[12] Gennisson JL, Catheline S, Chaffai S, Fink M. Transient elastography in anisotropic medium: Application to the measurement of slow and fast shear wave speeds in muscles. J Acoust Soc Am 2003; 114: 536-541

[13] Eby SF, Song P, Chen S, Chen Q, Greenleaf JF, An KN. Validation of shear wave elastography in skeletal muscle. J biomech 2013; 46: 2381-2387

[14] Morrow DA, Haut Donahue TL, Odegard GM, Kaufman KR. Transversely isotropic tensile material properties of skeletal muscle tissue. J Mech Behav Biomed Mater 2010; 3: 124-129

[15] Niitsu M, Michizaki A, Endo A, Takei H, Yanagisawa O. Muscle hardness measurement by using ultrasound elastography: A feasibility study. Acta Radiol 2011; 52: 99-105

[16] Kuo WH, Jian DW, Wang TG, Wang YC. Neck muscle stiffness quantified by sonoelastography is correlated with body mass index and chronic neck pain symptoms. UMB 2013; 39: 1356-1361

[17] Chang S, Kim MJ, Kim J, Lee MJ. Variability of shear wave velocity using different frequencies in acoustic radiation force impulse (ARFI) elastography: A phantom and normal liver study. Ultraschall in Med 2013; 34: 260-265

[18] Ewertsen C, Carlsen JF, Christiansen IR, Jensen JA, Nielsen MB. Evaluation of healthy muscle tissue by strain and shear wave elastography - Dependency on depth and ROI position in relation to underlying bone. Ultrasonics 2016; 71: 127-133

[19] Kot BC, Zhang Z], Lee AW, Leung VY, Fu SN. Elastic modulus of muscle and tendon with shear wave ultrasound elastography: Variations with different technical settings. PloS One 2012; 7: e44348 